\title{
A Primer of a Sensor Calibration Procedure for Embedded Applications
}

\author{
Igor Janevski \\ Faculty of Natural Sciences and \\ Mathematics \\ Ss. Cyril and Methodius University \\ 1000 Skopje, R. Macedonia
}

\author{
Roman Golubovski \\ Faculty of Natural Sciences and \\ Mathematics \\ Ss. Cyril and Methodius University \\ 1000 Skopje, R. Macedonia
}

\author{
Vanco Cabukovski \\ Faculty of Natural Sciences and \\ Mathematics \\ Ss. Cyril and Methodius University \\ 1000 Skopje, R. Macedonia
}

\begin{abstract}
Modern higher education is today supported by electronic Learning Management Systems providing formal educational learning material, some even integrating repositories containing supplemental material and providing adaptation during the learning process. Virtual laboratories allow access to rare or distributed equipment to students.

This paper depicts a primer of such fundamental supplement for students of instrumentation or embedded courses. The exercise provides theoretical background and a cloud access to an affordable hardware via SaaS (Software as a Service) as PaaS (Platform as a Service), or a web based access to a simulation engine. Either case, students are able to calculate and organize experiments, provide input values and get acquired or simulated result.
\end{abstract}

\section{General Terms}

eLearning, Adaptability, Agent-based, Learning Management Systems, Virtual Laboratory, Online Laboratory.

\section{Keywords}

Embedded Systems, NTC Probe, Data Acquisition, Instrumentation.

\section{INTRODUCTION}

The application aspects of the computer sciences are fundamental in the real-life computer based implementations. One of these is the computer systems interaction with the real/external world. Modern trends in computerized automation involve use of sensors and actuation, and the computer systems and embedded applications need to acquire physical phenomena in order to decide/calculate for some external response. Crucial aspect of the data acquisition in order to comprehend the real physical value is a calibration of sensory output. The practical aspects of the technical engineering disciplines require accessible laboratory tools not only for educational purposes, but also for training and validation of development activities in the industry.

Contemporary higher education is more or less supported by electronic Learning Management Systems (eLMS) providing formal educational learning material (text books, lectures, exercises, etc.). Newer eLMS even integrate CMS (Content Management System) repositories containing supplemental material (multimedia, books, articles, journals, etc.) to provide adaptation to individual student needs for steeper learning curves. Such AeLMS (Adaptive eLMS) have proved to be very successful in improving overall University education quality [1,2]. Some efforts in this field are Virtual Laboratories to make lab equipment and simulation engines accessible to students in cases when such is remote or in deficit $[3,4,5,6]$.
At the Faculty of Natural Sciences and Mathematics with the University Ss. Cyril and Methodius - Skopje a multi-agent AeLMS is developed in support of the formal curricula. This integrated IABUIS (Intelligent Agent-Based University Information System) is intended to achieve more effective learning curve by tailoring courses' curriculum to individual students' specific preferences, by following their activities in the regular eLMS containing the formal lecturing material and in the complementary informal CMS (Content Management System) containing carefully gathered supplement material (multimedia supplements) [7].

An appropriate supplement and corresponding virtual lab tool were added to the IABUIS AeLMS, and there is ongoing evaluation of student activities with respect to their usefulness. The AeLS is able to keep track of usage and to accommodate content automatically for each of the users, for the best learning result, which in turn is supported by a student model built from student's goals, preferences, and knowledge.

This paper presents a general mathematically based methodology of interfacing a computer system with sensors that need to be excited and calibrated according to vendor's specification. This is the general approach in the instrumentation, often used in developing the educational tools in the virtual laboratories $[8,9,10,11]$.

The educational tool presented in the paper depicts the process of proper interfacing a computer system and embedded application with a NTC (Negative Temperature Coefficients) thermal probe for measuring the environmental temperature. The process begins with designing a basic electronic circuit for required current excitation of the NTC probe. Than the voltage produced over its resistor is acquired via an A/D (Analog-to-Digital) converter - it is sampled and digitized. It is also calibrated for the pre amplification and finally a set of exponential equations are used to determine key parameters of voltage calibration in order to convert it into a temperature value. Decisions can be made consistent with the embedded application - the home automation in this particular case study. Data used for modeling of the resistance-temperature dependence is issued by the probe's manufacturer, and mathematical equations are already well known relations in physics.

\section{TEMPERATURE CALCULATION} 2.1 The Sensing Element

Just like with any other sensing, temperature measurement requires a transducing element that converts the environmental temperature to another physical phenomenon that can be sensed by a computer system. Such physical phenomenon is voltage which can be directly measured by 
available hardware. Since voltage is formed over a resistor when the current flows through it, and all materials change their resistance under temperature changes, the easiest way to convert temperature is by using an element with a temperature dependent variable resistance that modulates its voltage when excited with a constant current presented by the Ohm's Law $(V=R \cdot I)$.

Different materials (resistors or semiconductors) react differently on temperature changes - some raise their resistance with raising temperature like PTC (positive temperature coefficient) thermistors, or lower it like NTC thermistors. In general, their R-T dependence is nonlinear as shown in Figure 1 and sufficiently precise mathematical formula is required to model it.

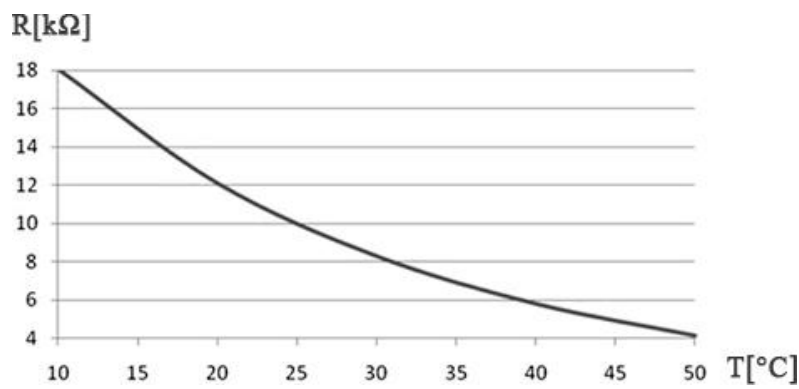

Fig 1: A typical NTC R-T curve $\left(10 \mathrm{k} \Omega @ 25^{\circ} \mathrm{C}\right)$

\subsection{The Mathematical Model}

The most precise model for NTC probes is the Reinhart-Stein relation [12]:

$$
\frac{1}{\mathrm{~T}}=\mathrm{A}+\mathrm{B}(\ln R)+C(\ln R)^{3}
$$

Here, the $T$ and $R$ parameters denote the relating temperature and resistance, and $A, B$ and $C$ are coefficients shaping the curve. The curve itself is usually provided in a tabular form by the vendors as shown in Figure 3 (minimal, maximal and average resistance values for every temperature).

Under these circumstances the temperature measurement process relies on measurement of $R$ and then on calculation of $T$, so what is left to be determined are the curve coefficients $A$, $B$ and $C$. These coefficients can be calculated by forming a system of three linear equations given bellow:

$$
\left\{\begin{array}{l}
\frac{1}{\mathrm{~T}_{1}}=\mathrm{A}+\mathrm{B}\left(\ln R_{1}\right)+C\left(\ln R_{1}\right)^{3} \\
\frac{1}{\mathrm{~T}_{2}}=\mathrm{A}+\mathrm{B}\left(\ln R_{2}\right)+C\left(\ln R_{2}\right)^{3} \\
\frac{1}{\mathrm{~T}_{3}}=\mathrm{A}+\mathrm{B}\left(\ln R_{3}\right)+C\left(\ln R_{3}\right)^{3}
\end{array}\right.
$$

The three pairs $\left(T_{i}, R_{i}\right)$ for $\mathrm{i}=1,2,3$ respectively can be taken from the R-T table (the framed pairs on Figure 2). Usually the pairs are chosen to hit the boundaries of the required measurement range and its mid-point to minimize the nonlinearity deviation and maximize the precision.
As soon as coefficients $A, B$ and $C$ are determined, the Steinhart-Hart formula can be directly implemented in the temperature measurement process.

\section{RESISTANCE MEASUREMENT}

Once the embedded application is calibrated for the particular NTC probe, the applications can use the temperature readings $\mathrm{T}$ in their control loops by simply measuring its resistance $\mathrm{R}$ periodically.

Since the resistance is not measurable directly and only the voltage is, a mathematical voltage-resistance relationship is required to be set up allowing resistance calculation of measured voltage. Computer systems can "measure" voltage through electronic circuits called ADC (analog-to-digital convertor) which sample the input voltage at some rate (samples/second) and digitize those samples with some precision (bits/range) [13].

\subsection{Voltage Modulation with Resistance}

To allow a modulation of the voltage over the NTC resistor $\left(R_{N T C}\right)$ it needs to be combined with another fixed resistor $(R)$ into a voltage divider [14]. The voltage divider between two resistors is presented in Figure 2.

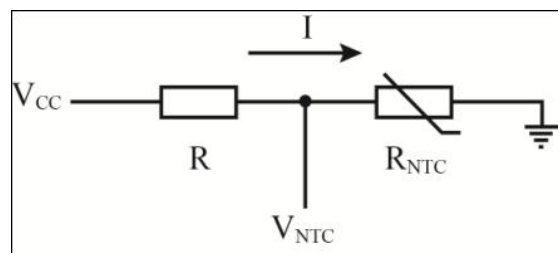

Fig 2: A voltage divider between two resistors

A constant $V_{C C}$ voltage is supplied to the divider which draws the current $I$ by the following formula:

$$
I=\frac{V_{C C}}{R+R_{N T C}}
$$

The variable voltage $V_{N T C}$ acquired over the probe can be calculated by the following relation:

$$
V_{N T C}=I \cdot R_{N T C}=V_{C C} \cdot \frac{R_{N T C}}{R+R_{N T C}}
$$

Now $R_{N T C}$ can be obtained directly from the measured $V_{N T C}$ and the known $R$ and $V_{C C}$ as follows:

$$
\begin{aligned}
& V_{N T C} \cdot R+V_{N T C} \cdot R_{N T C}=V_{C C} \cdot R_{N T C} \\
& R_{N T C}\left(V_{C C}-V_{N T C}\right)=R \cdot V_{N T C} \\
& R_{N T C}=R \cdot \frac{V_{N T C}}{V_{C C}-V_{N T C}}
\end{aligned}
$$

\subsection{Choosing the dividing resistor $R$}

Another precision related issue is the additional mathematical background for calculation of the optimal value of $R$, to achieve maximum measurement accuracy at lowest "cost" (dissipation) 


\begin{tabular}{|c|c|c|c|c|c|c|c|}
\hline & \multicolumn{2}{|c|}{$\mathrm{R}_{25}=10000 \Omega: \pm 3 \%$} & \multicolumn{4}{|c|}{$\mathrm{B}_{25 / 50}=3380 \mathrm{~K}\left(\mathrm{~B}_{25 / 85}=3435 \mathrm{~K}\right): \pm 1 \%$} & \\
\hline$\left({ }^{\circ} \mathrm{C}\right)$ & & $(\Omega)$ & & & & \multicolumn{2}{|c|}{$\left({ }^{\circ} \mathrm{C}\right)$} \\
\hline & & & & $\triangle \mathrm{R}$ & $-\triangle R$ & $\triangle \mathrm{T}$ & $-\triangle \mathrm{T}$ \\
\hline-55 & 408305.000 & 437139.000 & 467587.000 & 6.965 & 6.595 & 2.828 & 2.678 \\
\hline-54 & 385208.000 & 412169.000 & 440619.000 & 6.902 & 6.541 & 2.782 & 2.637 \\
\hline-53 & 363546.000 & 388762.000 & 415354.000 & 6.840 & 6.486 & 2.738 & 2.596 \\
\hline-52 & 343221.000 & 366814.000 & 391677.000 & 6.777 & 6.432 & 2.694 & 2.556 \\
\hline-51 & 324144.000 & 346226.000 & 369480.000 & 6.716 & 6.377 & 2.650 & 2.516 \\
\hline-50 & 306232.000 & 326906.000 & 348662.000 & 6.655 & 6.324 & 2.607 & 2.478 \\
\hline-49 & 289408.000 & 308770.000 & 329131.000 & 6.594 & 6.270 & 2.565 & 2.439 \\
\hline-48 & 273600.000 & 291739.000 & 310800.000 & 6.533 & 6.217 & 2.523 & 2.401 \\
\hline-47 & 258742.000 & 275740.000 & 293591.000 & 6.473 & 6.164 & 2.482 & 2.364 \\
\hline-46 & 244772.000 & 260706.000 & 277428.000 & 6.413 & 6. 111 & 2. 442 & 2.327 \\
\hline-45 & 231632.000 & 246574.000 & 262243.000 & 6.354 & 6.059 & 2.402 & 2.290 \\
\hline-44 & 219269.000 & 233284.000 & 247971.000 & 6.295 & 6.007 & 2.363 & 2.255 \\
\hline-43 & 207633.000 & 220782.000 & 234553.000 & 6.237 & 5.955 & 2.324 & 2.219 \\
\hline-42 & 196677.000 & 209018.000 & 221934.000 & 6.179 & 5.904 & 2.286 & 2.184 \\
\hline-41 & 186359.000 & 197945.000 & 210062.000 & 6. 121 & 5.853 & 2.248 & 2.150 \\
\hline-40 & 176638.000 & 187518.000 & 198889.000 & 6.063 & 5.802 & 2.211 & 2.116 \\
\hline-39 & 167476.000 & 177696.000 & 188370.000 & 6.006 & 5.751 & 2.175 & 2.082 \\
\hline-38 & 158839.000 & 168442.000 & 178465.000 & 5.950 & 5.700 & 2. 139 & 2.049 \\
\hline-37 & 150694.000 & 159720.000 & 169133.000 & 5.893 & 5.650 & 2.103 & 2.017 \\
\hline : & : & : & : & : & $:$ & $:$ & : \\
\hline 116 & 618.727 & 655.483 & 693.798 & 5.845 & 5.607 & 2.230 & 2.140 \\
\hline 117 & 604.055 & 640.092 & 677.669 & 5.870 & 5.629 & 2.252 & 2.159 \\
\hline 118 & 589.797 & 625.131 & 661.985 & 5.895 & 5.652 & 2.273 & 2.179 \\
\hline 119 & 575.937 & 610.585 & 646.734 & 5.920 & 5.674 & 2.294 & 2.199 \\
\hline 120 & 562.465 & 596.441 & 631.901 & 5.945 & 5.696 & 2.316 & 2.219 \\
\hline 121 & 549.366 & 582.688 & 617.474 & 5.969 & 5.718 & 2.337 & 2.239 \\
\hline 122 & 536.630 & 569.311 & 603.439 & 5.994 & 5.740 & 2.359 & 2.259 \\
\hline 123 & 524.245 & 556.301 & 589.785 & 6.019 & 5.762 & 2.381 & 2.279 \\
\hline 124 & 512.200 & 543.644 & 576.499 & 6.043 & 5.783 & 2.403 & 2.299 \\
\hline 125 & 500.484 & 531.331 & 563.571 & 6.067 & 5.805 & 2.425 & 2.320 \\
\hline
\end{tabular}

Fig 3: R-T table for a particular NTC probe

The primary concern is the A/D conversion precision by spanning the $V_{N T C}$ to the whole input range of the ADC. This allows for the smallest change in voltage to be sensed. For example, a 12-bit $\mathrm{ADC}$ with $5 \mathrm{~V}$ input range can sense $2^{12}$ (4096) discrete values with step of $1.22 \mathrm{mV}$. The idea is to map the $V_{N T C}$ to the input range $V_{A D C}$ as close as possible, and to have in mind the gain of that amplification for the temperature conversion. Firstly, the measuring range is defined ( $T_{M I N}$ and $T_{M A X}$ ), and then the corresponding max/min $R_{N T C}$ values marking the expected resistance span. Now the expected current $I$ can be calculated, and by knowing the $V_{C C}$ $R$ can be calculated by using the above formulas.

However, current $I$ is also subject to limitation with regard to the source. Furthermore, $R$ is obviously estimated with regard to $R_{N T C}$ and:

- lower resistors mean higher $I$, higher accuracy but also higher dissipation,

- $\quad$ higher $t$ resistors mean lower $I$, lower dissipation but also lower accuracy

So all these criteria need to be considered for estimating the optimal value for $R$.

\section{THE TOOL APPLICATION}

Implementations of computerized measurements and control range from home entertainment to heavy industries and space exploration. Home air-conditioning is a one popular application that can demonstrate the use of the above depicted instrumentation for digital temperature measurement.

In Figure 4 an embedded application using affordable Arduino controller [15] is shown. An NTC probe via a voltage divider on a breadboard is connected to one of its analog inputs. The air-conditioning system is controlled through one of Arduino's digital outputs protected with a relay.

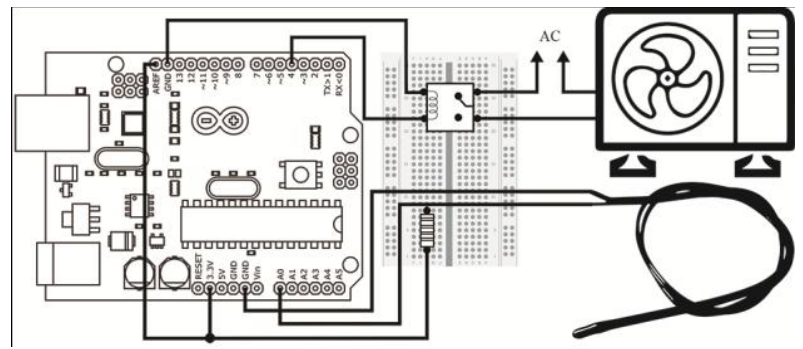

Fig 4: A simple home air conditioning system controller

The controlling algorithm for cooling is presented in Figure 5. The embedded software constantly monitors the environmental temperature $T$ and compares it to the set point $S P$ (desired temperature). If $T$ is higher than $S P$ the controller switches ON the compressor to start cooling, if $T$ is lower or equal to the $S P$ the compressor remains OFF. This ON/OFF switching can be controlled by a single digital output line. 


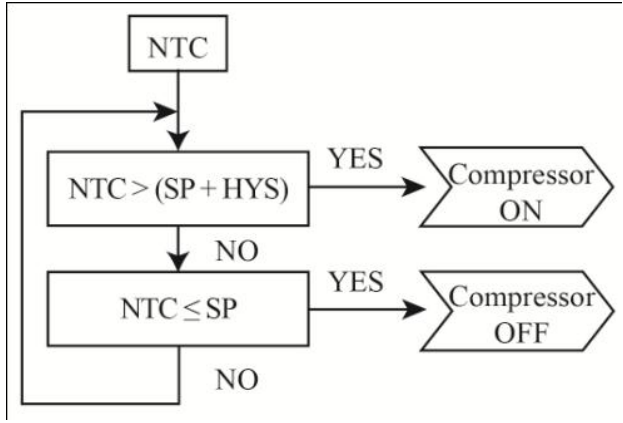

Fig 5: The hysteresis control flowchart

The hysteresis HYS is involved for practical reasons, to avoid frequent switching $\mathrm{ON}$ and $\mathrm{OFF}$ in case of minor variations of $T$. This saves both the controller electronics and the compressor health. HYS is de facto an offset in a temperature within which the controller would tolerate deviations of $T$ with the respect to $S P$. So now the algorithm switches ON the compressor if $T$ is higher than $S P+H Y S$, and switches OFF the compressor when $T$ goes below $S P$.

Controlling by hysteresis is a standard procedure in temperature control related applications, and similar mathematical approaches are using it in other data acquisition and control related applications.

The cloud service supporting this tool (accessible within the private university cloud) allows the following two usage scenarios:

1. A tool for educational experiments (SaaS) - students can use it to get acquainted to all the aspects of building automation for temperature control.

2. A development environment (PaaS) - students can use it to develop new applications (tools) and implement data acquisition and control algorithms.

The developed and validated tools as SaaS can be used also by graduates involved in the industry.

\section{CONCLUSION}

Mathematical modeling and formulations are crucial foundation to the computerized interaction with our reality, and to the technological implementations. This demonstration of data acquisition and control application depicts the power of applicative implementations in solving real life problems with the use of computer.

The embedded demo application needs to perform three stages of calculations - (1) probe calibration, (2) signal conditioning, and (3) control application with discrete logic.

Further efforts are being made to provide additional material on different or concurrent methodologies and implemented technologies to provide comparative studies on modern instrumentation and signal conditioning as well as on the latest of the embedded design.

Latest internet technologies like cloud computing and SaaS/PaaS directly support the educational process, by providing students' access to learning resources through the virtual laboratories concept. This primer of a sensor calibration procedure for embedded applications is a prominent example of how technical engineering students are supported with educational tool for the instrumentation, data acquisition and general embedded systems courseware.

\section{REFERENCES}

[1] Cabukovski, V. 2011 "IABUIS - An Intelligent AgentBased University Information System, Lecture Notes in Information technology", Information Engineering Research Institute, USA, Vol. 3-4, pp 13-19.

[2] Cabukovski, V., Golubovski, R., Temjanovski, R.. 2016. "Learning Repository Adaptability In An Agent Based University Environment", International Journal of Computer Science \& Information Technology (IJCSIT), Volume 8, No 3, pp. 103-118.

[3] Mercer, L., Prusinkiewicz, P., Hanan J. 1990 "Concept and design of a virtual laboratory", Proceedings on Graphics Interface '90, Nova Scotia, Canada, May 1990, pp. 149-155.

[4] Wen, F.. 2012 "Open Web-based Virtual Lab Experimental Enhanced Educational Environment", eLearning - Theories, Design, Software and Applications, ed Patrizia Ghislandi, InTech, pp. 239-260.

[5] Wen, F., Suo, D., Chen, M., and Li, J. 2010 "An OpenLab Platform with Virtual Experiment Learning System", 2010 2nd IEEE International Conference on Network Infrastructure and Digital Content, Beijing, China, September 2010, IEEE

[6] Rheingold, H. 1993 The Virtual Community: Homesteading on the Electronic Frontier. AddisonWesley, MA

[7] Cabukovski, V., Golubovski, R.. 2016 "An Adaptive Course Materials Selection into a Multi-Agent Based eLearning System", International Journal of Computer Applications, 144(8), pp. 4-8.

[8] Sell, R., Seiler, S., Ptasik, D.. 2012 "Embedded System and Robotic Education in a Blended Learning Environment Utilizing Remote and Virtual Labs in the Cloud, Accompanied by 'Robotic HomeLab Kit'", International Journal of Emerging Technologies in Learning (iJET), 7(4), pp. 25-33.

[9] Sell, R.. 2013 "Remote Laboratory Portal for Robotic and Embedded System Experiments", International Journal of Emerging Technologies in Learning (iJET), 9(8), pp. 23-26.

[10] Ma, J, Nickerson, J.V.. 2006 "Hands-On, Simulated, and Remote Laboratories: A Comparative Literature Review", ACM Computing Surveys, 38(3), art.7.

[11] Ali, H.A., El-Ghareeb H.A.. 2014 "Implementation of Cloud-based Virtual Labs for Educational Purposes", International Journal of Computer Science and Network Security (IJCSNS), 14(7), pp. 45-49.

[12] Online available at: https://en.wikipedia.org/wiki/Steinhart\%E2\%80\%93Har t_equation

[13] Northrop, R. B.. 2005 Introduction to Instrumentation and Measurements, Taylor \& Francis.

[14] Bird, J.. 2009 Electrical and Electronic Principles and Technology, Elsevier, Newnes.

[15] Online available at: https://www.arduino.cc/ 